\title{
Prevalence and correlates of anaemia in adolescents in Riyadh city, Kingdom of Saudi Arabia
}

\author{
Al-Johara M Alquaiz ${ }^{1,2}$, Tawfik AM Khoja ${ }^{3}$, Abdullah Alsharif ${ }^{4}$, Ambreen Kazi ${ }^{1, *}$, \\ Ashry Gad Mohamed ${ }^{2}$, Hamad Al Mane ${ }^{5}$, Abdullah Aldiris ${ }^{6}$ and Shaffi Ahamed Shaikh ${ }^{2}$ \\ 'Princess Nora Bint Abdullah Chair for Women's Health Research, Research Chair Program, King Saud University, \\ PO Box 231831, Riyadh 1 1321, Kingdom of Saudi Arabia: ${ }^{2}$ Department of Family \& Community Medicine, College \\ of Medicine, King Saud University, Riyadh, Kingdom of Saudi Arabia: ${ }^{3}$ Gulf Ministry of Health, Riyadh, Kingdom of \\ Saudi Arabia: ${ }^{4}$ Council of Cooperative Health Insurance, Ministry of Health, Riyadh, Kingdom of Saudi Arabia: \\ ${ }^{5}$ Ministry of Health, Riyadh, Saudi Arabia: ${ }^{6}$ Central Hospital Laboratory, Ministry of Health, Riyadh, Kingdom of \\ Saudi Arabia
}

Submitted 22 October 2014: Final revision received 12 February 2015: Accepted 11 March 2015: First published online 4 May 2015

\begin{abstract}
Objective: To determine the prevalence and correlates of anaemia in male and female adolescents in Riyadh, Kingdom of Saudi Arabia.

Design: A cross-sectional community-based study.

Setting: Five primary health-care centres in Riyadh.

Subjects: We invited 203 male and 292 female adolescents aged 13-18 years for interview, anthropometric measurements and complete blood count. Blood $\mathrm{Hb}$ was measured with a Coulter Cellular Analysis System using the light scattering method. Results: Using the WHO cut-off of $\mathrm{Hb}<12 \mathrm{~g} / \mathrm{dl}, 16 \cdot 7 \%$ ( $n$ 34) of males and $34.2 \%$ ( $n$ 100) of females were suffering from anaemia. Mean $\mathrm{Hb}$ in males and females was 13.5 (SD 1.4) and 12.3 (SD 1.2) g/dl, respectively. Values for mean cell volume, mean cell $\mathrm{Hb}$, mean corpuscular $\mathrm{Hb}$ concentration and red cell distribution width in male and female adolescents were 77.8 (SD 6.2) v. 76.4 (SD 10.3) $\mu \mathrm{m}^{3}, 26 \cdot 1$ (SD 2.7) v. 25.5 (sD 2.6) pg, 32.7 (sD 2.4) v. 32.2 (sD 2.6) g/dl and 13.9 (SD 1.4) v. 13.6 (SD 1.3) \%, respectively. Multivariate logistic regression revealed that a positive family history of Fe-deficiency anaemia (OR $=4.7 ; 95 \%$ CI 1.7, 12.2), infrequent intake $(\mathrm{OR}=3.7$; $95 \%$ CI $1.3,10 \cdot 0)$ and never intake of fresh juices (OR $=3.5 ; 95 \%$ CI $1.4,9.5)$ and being $13-14$ years of age $(\mathrm{OR}=3 \cdot 1 ; 95 \% \mathrm{CI} 1 \cdot 2,9 \cdot 3)$ were significantly associated with anaemia in male adolescents; whereas in females, family history of Fe-deficiency anaemia (OR=3.4; $95 \%$ CI 1.5, 7.6), being overweight (OR=3.0; $95 \%$ CI 1.4, 6.1), no intake of fresh juices (OR $=2 \cdot 6$; $95 \%$ CI $1 \cdot 4,5 \cdot 1$ ), living in an apartment $(\mathrm{OR}=2 \cdot 0 ; 95 \% \mathrm{CI} 1 \cdot 1,3 \cdot 8)$ and living in a small house $(\mathrm{OR}=2 \cdot 5 ; 95 \% \mathrm{CI}$ $1 \cdot 2,5 \cdot 3)$ were significantly associated with anaemia.

Conclusions: Anaemia is more prevalent among Saudi female adolescents as compared with males. Important factors like positive family history of Fe-deficiency anaemia, overweight, lack of fresh juice intake and low socio-economic status are significantly associated with anaemia in adolescents.
\end{abstract}

Anaemia is global public health problem affecting both developed and developing countries with major consequences for human health as well as social and economic development. According to the WHO, $50 \%$ of the world's population is below 25 years of age and almost one-third is 10-24 years old. Globally, the prevalence of anaemia among adolescents is $6 \%$ in developed countries and $27 \%$ in developing countries ${ }^{(1)}$.

Regional studies on anaemia in adolescents have reported varying results. A study on Kuwaiti adolescent girls (aged $17-24$ years) found $30 \%$ of females to be suffering from anaemia $^{(2)}$. A study conducted in high and low socioeconomic areas of Baghdad province in Iraq found $12.9 \%$ and $17 \cdot 6 \%$ of male and female adolescents (aged 11-19 years) to be suffering from anaemia ${ }^{(3)}$. In contrast, a study from the United Arab Emirates reported Fe-deficiency anaemia (IDA) prevalence of 5\% among adolescents (15-18-year-old males and females) ${ }^{(4)}$.

In the Kingdom of Saudi Arabia (KSA), most of the studies on nutritional status have focused on children and adults, hence data on the nutritional status of adolescents are meagre $^{(5,6)}$. One cross-sectional survey conducted by the 
Health Directorate in Riyadh city among schoolgirls (16-18 years old) showed that the prevalence of anaemia was $40 \cdot 5 \%^{(7)}$. A study conducted in four provinces of KSA found that anaemia was prevalent at moderate to high rates among adults, ranging from $7 \cdot 2$ to $16.5 \%$ in the males and from 10.8 to $23.5 \%$ in the females; and from 16.5 to $41.3 \%$ in children $(<14 \text { years })^{(8)}$. Abalkhail et al. conducted a study in Jeddah schools in 2002 and found $20.5 \%$ of Saudi students ( $<12$ years) suffering from anaemia. Anaemia was significantly more prevalent among 12 years old, those attending governmental schools, born to loweducated mothers and belonging to low socio-economic background $^{(9)}$.

Research has found that adolescents are at an increased risk of developing anaemia due to increasing Fe demand during puberty, menstrual losses, limited dietary Fe intake and faulty dietary habits ${ }^{(10)}$. Deficiencies of other nutrients (such as folic acid, protein, vitamin $\mathrm{B}_{12}$, vitamin $\mathrm{A}$ and $\mathrm{Cu}$ ) and poor absorption of $\mathrm{Fe}$ from diets high in phytate or phenolic compounds may contribute ${ }^{(11)}$. Additionally, household characteristics such as hygiene, parental education, household income and number of children in the family are also important determinants of anaemia ${ }^{(12)}$. Recently, lifestyle factors leading to obesity such as sedentary lifestyle, fast-food intake and preference for indoor activities have been associated with anaemia ${ }^{(13)}$.

Anaemia, especially in females, affects the whole life cycle if not diagnosed or treated in a timely manner. It could lead to poor maternal and neonatal outcomes and there is increased risk of disabilities in the post-reproductive period $^{(14)}$. Newborns or young children born to anaemic mothers are more likely to have atrial septal defects, attention deficit hyperactivity disorder, delayed development and mental retardation ${ }^{(14)}$. Clinical studies also support findings that IDA is significantly associated with increased risks of unipolar and bipolar depressive disorder and anxiety disorders $^{(15)}$. Additionally, Fe can have bone-protective effects and so deficiency of this element can negatively affect bone mass, which is especially important during infancy and adolescence $^{(16)}$. Anaemia not only affects the health profile but also hampers educational achievement and causes differences in performance of both sexes ${ }^{(17)}$; hence it is pertinent to identify the significant factors associated with anaemia among adolescents.

KSA not only has a high prevalence of anaemia but factors like unawareness, obesity and unhealthy lifestyle are highly prevalent ${ }^{(18)}$. In the absence of current data, the present study was designed with the objective of measuring the prevalence of anaemia and its correlates in Saudi male and female adolescents in Riyadh city, KSA.

\section{Methods}

We conducted a community-based, two-staged household cross-sectional survey. According to the 2010-2011 census report, Riyadh has a total population of 3151209 individuals ${ }^{(19)}$. In the first stage a complete list of primary health-care centres (PHCC) was formulated and one PHCC was randomly selected from each of the five administrative regions (East, West, North, South and Central) of Riyadh city. In the second stage, households were randomly selected and invited from the catchment area of each selected PHCC. Inclusion criteria for participants were Saudi national, aged between $\geq 13$ and $\leq 18$ years and resident of Riyadh city, KSA. Assuming that an average Saudi family in Riyadh region will have at least one eligible adolescent in their house, and keeping the refusal rate at $20 \%$, we planned to select 110 houses from the catchment area of each PHCC making a total sample of 550 adolescents. If more than one eligible adolescent was present in a house, all those fulfilling the inclusion criteria were invited to participate and visit the respective section in the PHCC (there is separate section in the PHCC for males and females) for questionnaire filling, anthropometric measurements and blood sample collection. On behalf of adolescents, their parents read and signed the informed consent. The Ethical Review Board of King Saud University approved the study protocol.

\section{Tool for data collection}

The questionnaires inquired about sociodemographic characteristics (age, education, type and ownership of residence); dietary history (frequency of intake of red meat, vegetables, eggs, tea or coffee, fruit juices, beverages and laban (diluted yoghurt commonly used in KSA)); past or current medical conditions (peptic ulcer, haemorrhoids, cancer, bilharziasis and blood transfusion); and family history of IDA and smoking history. In addition, for females, specific questions were asked about age at menarche, menstrual flow and type (passing any blood clots). Information on number of pregnancies, abortions and use of contraceptives was collected from married adolescents. Male and female nurses were trained to conduct the interviews, take anthropometric measurements and draw, label and store blood samples. Weight and height were measured using an electronic scale (Seca 220, Hamburg, Germany, 2009). Based on the formula weight $/$ height $^{2}$, BMI $\left(\mathrm{kg} / \mathrm{m}^{2}\right)$ was calculated for each participant.

\section{Blood sample for $\mathrm{Hb}$ estimation}

A $5 \mathrm{ml}$ sample of venous blood was taken for haematological examinations including $\mathrm{Hb}$, red blood cell count, haematocrit and red cell indices: mean cell volume (MCV), mean cell $\mathrm{Hb}(\mathrm{MCH})$, mean cell $\mathrm{Hb}$ concentration (MCHC) and red blood cell distribution width (RDW). Blood samples were collected in labelled and prepared tubes containing EDTA or heparin and stored in the refrigerator. By the end of the day (16.00 hours) all samples were sent in a cold box to the King Khalid Hospital laboratory where $\mathrm{Hb}$ estimation was performed by laboratory technicians 
using the UniCel ${ }^{\circledR}$ DxH $^{\mathrm{TM}} 800$ Coulter $^{\circledR}$ Cellular Analysis System (Beckman Coulter, Inc., USA). The light scattering method was used to measure $\mathrm{Hb}$ and red cell indices under the supervision of a haematologist. Quality control procedures (Coulter ${ }^{\circledR}$ 6C Cell Control) as instructed by the manufacturer were followed.

\section{Statistical analysis}

Data were analysed using the statistical software package IBM SPSS Statistics version 19. The outcome variable (anaemia) was dichotomized as anaemic and non-anaemic according to the WHO cut-off of $\mathrm{Hb}$ level $<12 \mathrm{~g} / \mathrm{dl}^{(20)}$. Descriptive statistics with means and standard deviations were calculated for blood indices, whereas proportions were calculated for categorical variables. In order to formulate categorical variables, age was divided into three categories: $13-14$ years, $15-16$ years and $17-18$ years. BMI was divided into the following categories: underweight $\left(\leq 18.5 \mathrm{~kg} / \mathrm{m}^{2}\right)$, normal $\left(>18.5-24.9 \mathrm{~kg} / \mathrm{m}^{2}\right)$, overweight $\left(\geq 25 \cdot 0-29.9 \mathrm{~kg} / \mathrm{m}^{2}\right)$ and obese $\left(\geq 30 \cdot 0 \mathrm{~kg} / \mathrm{m}^{2}\right)$. Similarly, dietary intake was divided into: frequent $(5-7 \mathrm{~d} /$ week), infrequent (2-4 d/week) and no intake (0-1 d/week). The level of statistical significance was kept at $P<0 \cdot 05$. Univariate analysis was done to identify biologically plausible and statistically significant variables. Forward multiple logistic regression analyses were performed, using the stepwise forward method, to identify important correlates associated with anaemia in male and female adolescents. Plausible interactions were checked before developing the final model.

\section{Results}

Initially we planned to invite 550 households; however, we were able to achieve our sample size by enrolling 347 households. All of them verbally agreed to come and participate, but fifty households did not report to the centre during the data collection period. With response rate of $85 \%, 297$ households participated; 161 adolescents belonged to the 'single adolescent per family' category, 188 adolescents belonged to 'two adolescents per family', whereas the rest $(n 151)$ belonged to 'three or more adolescents per family'. Five forms were not included in the analysis because of incomplete information/missing blood reports; hence in total there were 495 participants.

There was no significant difference between the mean age of males and females (15.3 (SD 1.7) v. 15.2 (SD 1.7) years, respectively; $P=0.92$ ), ranging from 13 to 18 years. Most (about $65-75 \%$ ) of the male and female adolescents were studying either in secondary or intermediate level $(P=0 \cdot 14)$. Few adolescents $(2-8 \%)$ were living in shared residences as compared with rented and self-owned accommodation. Mean BMI for males and females was 22.1 (SD 5.71) and $22.2(\mathrm{SD} 5.71) \mathrm{kg} / \mathrm{m}^{2}$, respectively $(P=0 \cdot 86$; Table 1$)$. Only $3.9 \%(n 8)$ of male adolescents mentioned that they were current smokers and smoked $<5$ cigarettes/d (none of the females was a smoker). Menstrual history was taken from females. Only a few (7.5\%) adolescent females complained about passing clots and $9.5 \%$ mentioned heavy periods; however, their mean $\mathrm{Hb}$ was within the normal range (12.4 (sD 1.3) g/dl). Among females, $3.4 \%$ ( $n 10)$ were married, five were pregnant and two were lactating, and their mean $\mathrm{Hb}$ was $12 \cdot 1$ (SD 1.4) g/dl. None of the adolescents were using any type of contraceptive (results not shown in table). More male anaemic adolescents were suffering from intestinal worms $(8.8 \% v \cdot 7 \cdot 0 \%)$, bilharziasis $(11.8 \% v .7 \cdot 0 \%)$ and family history of IDA (29.0\% $v .19 \cdot 0 \%)$ as compared with female anaemic adolescents. Only two participants among males and females mentioned having haemorrhoids.

Keeping the cut-off at $\mathrm{Hb}<12 \mathrm{~g} / \mathrm{dl}$, the prevalence of anaemia was $17.0 \%(34 / 203)$ in males and $34.2 \%$ (100/292) in female adolescents in Riyadh. Mean $\mathrm{Hb}$ in males and females was 13.5 (SD 1.8) and 12.4 (SD 1.5) mg/dl, respectively $(P=0 \cdot 00)$. Mean values for blood indices (MCH, MCV, MCHC and RDW) are mentioned in Table 2. The frequency and unadjusted odds ratio and $95 \%$ confidence interval for different dietary items in males and females are presented in Table 3. Fifty-three per cent of anaemic males and $52 \%$ of anaemic females consumed red meat frequently. Similarly, $65 \%$ of anaemic males and $68 \%$ of anaemic females consumed green vegetables frequently. Fewer anaemic females $(26 \%)$ than anaemic males (35\%) consumed tea frequently. Almost equal numbers of anaemic males (50\%) and anaemic females (54\%) consumed laban (diluted yoghurt) frequently. Fifty per cent of anaemic males and $44 \%$ of anaemic females were frequently consuming eggs. About $50 \%$ of anaemic males and females were not taking fruit juices at all.

Univariate analysis found young age (13-14 years; $\mathrm{OR}=3 \cdot 1 ; 95 \%$ CI $1 \cdot 2,8 \cdot 3)$, living in an apartment $(\mathrm{OR}=$ $2 \cdot 3 ; 95 \%$ CI $1 \cdot 1,5 \cdot 3)$, living in a shared house $(\mathrm{OR}=5 \cdot 0$; $95 \%$ CI $1 \cdot 1,20 \cdot 1$ ), having a past history of bilharziasis $(\mathrm{OR}=5 \cdot 5 ; 95 \% \mathrm{CI} 1.3,23 \cdot 2)$, having a positive family history of IDA (OR $=5.0 ; 95 \%$ CI $1.9,12.6)$ and infrequent and never intake of fresh juices $(\mathrm{OR}=3 \cdot 7 ; 95 \% \mathrm{CI} 1 \cdot 3$, $10 \cdot 1$ and $\mathrm{OR}=4 \cdot 3 ; 95 \%$ CI $1 \cdot 6,11 \cdot 2$, respectively) were significantly associated with anaemia among male adolescents (Tables 1 and 3). In females, univariate analysis found age group of 15-16 years was protective of anaemia $(O R=0.5 ; 95 \%$ CI $0.2,0.8)$. Similar to male adolescents, living in an apartment or a small house $(\mathrm{OR}=1 \cdot 9 ; 95 \% \mathrm{CI} 1 \cdot 1,3 \cdot 4$ and $\mathrm{OR}=2 \cdot 6 ; 95 \%$ CI 1.4, 5.1, respectively), having a family history of IDA (OR $=3.5$; $95 \%$ CI $1.6,7.6)$ and infrequent and no intake of fresh juices $(\mathrm{OR}=2 \cdot 3 ; 95 \% \mathrm{CI} 1 \cdot 2,4 \cdot 6$ and $\mathrm{OR}=3 \cdot 2 ; 95 \% \mathrm{CI} 1 \cdot 7$, $5 \cdot 9$, respectively) were significant in female adolescents (Tables 1 and 3 ). In contrast to males, overweight category (BMI between 25.0 and $29.9 \mathrm{~kg} / \mathrm{m}^{2}$ ) was significantly associated with anaemia in female adolescents $(\mathrm{OR}=2 \cdot 6$; $95 \%$ CI 1.3, 5·1; Table 1). 
Table 1 Univariate analysis showing unadjusted odds ratios and $95 \%$ confidence intervals for associations between sociodemographic characteristics, family history, health status and anaemia in male and female adolescents (aged 13-18 years) in Riyadh, Kingdom of Saudi Arabia ( $n$ 495)

\begin{tabular}{|c|c|c|c|c|c|c|c|c|c|c|c|c|}
\hline \multirow[b]{3}{*}{ Characteristic } & \multicolumn{6}{|c|}{ Males (n 203) } & \multicolumn{6}{|c|}{ Females ( $n$ 292) } \\
\hline & \multicolumn{2}{|c|}{$\begin{array}{c}\text { Anaemic } \\
(n 34,16.7 \%)\end{array}$} & \multicolumn{2}{|c|}{$\begin{array}{c}\text { Normal } \\
(n 169,83 \cdot 3 \%)\end{array}$} & \multicolumn{2}{|c|}{ Unadjusted } & \multicolumn{2}{|c|}{$\begin{array}{c}\text { Anaemic } \\
(n 100,34 \cdot 2 \%)\end{array}$} & \multicolumn{2}{|c|}{$\begin{array}{c}\text { Normal } \\
\text { (n 192, 65.8 \%) }\end{array}$} & \multicolumn{2}{|c|}{ Unadjusted } \\
\hline & $n$ & $\%$ & $n$ & $\%$ & OR & $95 \% \mathrm{Cl}$ & $n$ & $\%$ & $n$ & $\%$ & OR & $95 \% \mathrm{Cl}$ \\
\hline \multicolumn{13}{|l|}{ Age group } \\
\hline $17-18$ years & 6 & $17 \cdot 6$ & 51 & $30 \cdot 2$ & 1.0 & Ref. & 38 & 38 & 49 & $25 \cdot 5$ & 1.0 & Ref. \\
\hline $15-16$ years & 7 & $20 \cdot 6$ & 61 & $36 \cdot 1$ & 0.9 & $0.3,3.1$ & 23 & 23 & 62 & $32 \cdot 3$ & 0.5 & $0.2,0.8$ \\
\hline $13-14$ years & 21 & $61 \cdot 8$ & 57 & 33.7 & $3 \cdot 1$ & $1 \cdot 2,8 \cdot 3$ & 39 & 39 & 81 & $42 \cdot 2$ & 0.6 & $0.3,1 \cdot 1$ \\
\hline \multicolumn{13}{|c|}{ Educational level of participant } \\
\hline Secondary school & 5 & $14 \cdot 7$ & 38 & $22 \cdot 5$ & $1 \cdot 0$ & Ref. & 25 & 25 & 32 & $16 \cdot 7$ & 1.0 & Ref. \\
\hline Intermediate & 12 & $35 \cdot 3$ & 62 & $36 \cdot 7$ & 1.5 & $0.4,4.5$ & 43 & 43 & 94 & $49 \cdot 0$ & 0.6 & $0.3,1.1$ \\
\hline Elementary & 14 & $41 \cdot 2$ & 53 & 31.4 & $2 \cdot 0$ & $0.6,6.1$ & 24 & 24 & 57 & 29.7 & 0.6 & $0.2,1.1$ \\
\hline Illiterate & 3 & $8 \cdot 8$ & 16 & 9.5 & 1.4 & $0.3,6.7$ & 8 & 8 & 9 & 4.7 & $1 \cdot 1$ & $0.4,3.4$ \\
\hline \multicolumn{13}{|l|}{ Type of housing } \\
\hline Villa (modern house) & 13 & $38 \cdot 2$ & 97 & $57 \cdot 7$ & 1.0 & Ref. & 37 & 37 & 107 & 55.7 & 1.0 & Ref. \\
\hline Apartment & 14 & $41 \cdot 2$ & 45 & $26 \cdot 8$ & $2 \cdot 3$ & $1 \cdot 1,5 \cdot 3$ & 38 & 38 & 57 & $29 \cdot 7$ & 1.9 & $1 \cdot 1,3.4$ \\
\hline Small house & 7 & $20 \cdot 6$ & 27 & $15 \cdot 5$ & $2 \cdot 0$ & $0.7,5.5$ & 25 & 25 & 28 & $14 \cdot 6$ & $2 \cdot 6$ & $1 \cdot 4,5 \cdot 1$ \\
\hline \multicolumn{13}{|l|}{ Ownership of house } \\
\hline Self-owned & 19 & 55.9 & 124 & 73.4 & 1.0 & Ref. & 60 & 60 & 140 & $72 \cdot 9$ & 1.0 & Ref. \\
\hline Share & 3 & $8 \cdot 8$ & 4 & $2 \cdot 4$ & $5 \cdot 0$ & $1 \cdot 1,20 \cdot 1$ & 5 & 5 & 2 & 1.0 & $5 \cdot 8$ & $1 \cdot 1,30.9$ \\
\hline Rent & 12 & $35 \cdot 3$ & 41 & $24 \cdot 3$ & 1.9 & $0.8,4 \cdot 2$ & 35 & 35 & 50 & $26 \cdot 0$ & 1.6 & $1 \cdot 1,2 \cdot 7$ \\
\hline \multicolumn{13}{|l|}{ BMI category } \\
\hline Normal & 16 & $47 \cdot 1$ & 88 & $52 \cdot 1$ & 1.0 & Ref. & 45 & 45 & 109 & $56 \cdot 8$ & 1.0 & Ref. \\
\hline Underweight & 10 & 29.4 & 43 & 25.4 & 1.3 & $0.5,3.1$ & 23 & 23 & 45 & 23.4 & 1.2 & $0.6,2.3$ \\
\hline Overweight & 7 & $20 \cdot 6$ & 27 & $16 \cdot 0$ & 1.4 & $0.5,3 \cdot 8$ & 25 & 25 & 23 & $12 \cdot 0$ & 2.6 & $1 \cdot 3,5 \cdot 1$ \\
\hline Obese & 1 & 2.9 & 11 & 6.5 & 0.5 & $0.1,4.1$ & 7 & 7 & 15 & $7 \cdot 8$ & $1 \cdot 1$ & $0.4,2.9$ \\
\hline \multicolumn{13}{|l|}{ Intestinal worms } \\
\hline No & 31 & 91.2 & 161 & $95 \cdot 3$ & 1.0 & Ref. & 93 & 93 & 180 & 93.8 & 1.0 & Ref. \\
\hline Yes & 3 & $8 \cdot 8$ & 8 & $4 \cdot 7$ & 1.9 & $0 \cdot 4,7 \cdot 7$ & 7 & 7 & 12 & $6 \cdot 3$ & $1 \cdot 1$ & $0.4 \cdot 2 \cdot 9$ \\
\hline \multicolumn{13}{|l|}{ Bilharziasis } \\
\hline No & 30 & $88 \cdot 2$ & 165 & 97.6 & 1.0 & Ref. & 93 & 93 & 185 & $96 \cdot 4$ & 1.0 & Ref. \\
\hline Yes & 4 & $11 \cdot 8$ & 4 & $2 \cdot 4$ & 5.5 & $1 \cdot 3,23 \cdot 2$ & 7 & 7 & 7 & $3 \cdot 6$ & 1.9 & $0.6,5.8$ \\
\hline \multicolumn{13}{|c|}{ Family history of Fe-deficiency anaemia } \\
\hline No & 24 & $70 \cdot 6$ & 156 & $92 \cdot 3$ & 1.0 & Ref. & 81 & 81 & 180 & 93.8 & 1.0 & Ref. \\
\hline Yes & 10 & 29.4 & 13 & 7.7 & $5 \cdot 0$ & $1.9,12 \cdot 6$ & 19 & 19 & 12 & $6 \cdot 3$ & 3.5 & $1 \cdot 6,7 \cdot 6$ \\
\hline \multicolumn{13}{|c|}{ Family history of hereditary blood disorder } \\
\hline No & 27 & 84.4 & 131 & 77.5 & 1.0 & Ref. & 85 & 85 & 159 & $83 \cdot 2$ & 1.0 & Ref. \\
\hline Yes & 5 & $15 \cdot 6$ & 38 & 22.5 & 0.6 & $0 \cdot 2,1 \cdot 7$ & 15 & 15 & 33 & $16 \cdot 8$ & 0.8 & $0.4,1.7$ \\
\hline
\end{tabular}

Ref., reference category.

Table 2 Mean $\mathrm{Hb}$ and red blood cell indices in male and female adolescents (aged 13-18 years) in Riyadh city, Kingdom of Saudi Arabia (males, $n$ 203; females, $n$ 292)

\begin{tabular}{|c|c|c|c|c|c|c|}
\hline Variable & Mean & SD & Normal range & Minimum value & Maximum value & Interquartile value \\
\hline \multicolumn{7}{|c|}{ Hb level ( $\mathrm{g} / \mathrm{dl})$} \\
\hline Male & 13.5 & 1.4 & \multirow[t]{2}{*}{$12 \cdot 0-13 \cdot 0$} & 8.4 & $16 \cdot 7$ & $2 \cdot 0$ \\
\hline Female & $12 \cdot 3$ & 1.2 & & 7.4 & 14.9 & 1.9 \\
\hline \multicolumn{7}{|c|}{ Mean cell volume $\left(\mu m^{3}\right)$} \\
\hline Male & $77 \cdot 8$ & $6 \cdot 2$ & \multirow[t]{2}{*}{$80-94$} & $62 \cdot 7$ & $89 \cdot 0$ & $9 \cdot 6$ \\
\hline Female & $76 \cdot 4$ & $10 \cdot 3$ & & $60 \cdot 7$ & 89.9 & 8.3 \\
\hline \multicolumn{7}{|c|}{ Mean cell Hb (pg) } \\
\hline Male & $26 \cdot 1$ & $2 \cdot 7$ & \multirow[t]{2}{*}{$27-32$} & $20 \cdot 2$ & $32 \cdot 8$ & $4 \cdot 1$ \\
\hline Female & $25 \cdot 5$ & $2 \cdot 6$ & & $18 . \overline{5}$ & 31.0 & 3.9 \\
\hline \multicolumn{7}{|c|}{ Mean cell $\mathrm{Hb}$ concentration $(\mathrm{g} / \mathrm{dl})$} \\
\hline Male & $32 \cdot 7$ & $2 \cdot 4$ & \multirow[t]{2}{*}{$32-36$} & $27 \cdot 2$ & $38 \cdot 8$ & 3.1 \\
\hline Female & $32 \cdot 2$ & $2 \cdot 6$ & & 23.3 & 38.0 & 3.6 \\
\hline \multicolumn{7}{|c|}{ Red cell distribution width (\%) } \\
\hline Male & 13.9 & 1.4 & \multirow{2}{*}{$11.5-14.5$} & 12 & 18 & 1.8 \\
\hline Female & $13 \cdot 6$ & 1.3 & & 12 & 16 & 1.5 \\
\hline
\end{tabular}

Forward multivariate logistic regression revealed that having a family history of IDA (OR=4.7; $95 \%$ CI $1 \cdot 7,12 \cdot 2)$, infrequent and no intake of fresh juices $(\mathrm{OR}=3.7 ; 95 \% \mathrm{CI}$
$1 \cdot 3,10 \cdot 0$ and $\mathrm{OR}=3 \cdot 5 ; 95 \% \mathrm{CI} 1 \cdot 4,9 \cdot 5$, respectively) and young age $(\mathrm{OR}=3 \cdot 1 ; 95 \% \mathrm{CI} 1 \cdot 2,9 \cdot 3)$ were significantly associated with anaemia in male adolescents (after adjusting 
Table 3 Univariate analysis showing unadjusted odds ratios and $95 \%$ confidence intervals for associations between dietary items and anaemia in male and female adolescents (aged $13-18$ years) in Riyadh, Kingdom of Saudi Arabia ( $n$ 495)

\begin{tabular}{|c|c|c|c|c|c|c|c|c|c|c|c|c|}
\hline \multirow[b]{3}{*}{ Characteristic } & \multicolumn{6}{|c|}{ Males (n 203) } & \multicolumn{6}{|c|}{ Females ( $n$ 292) } \\
\hline & \multicolumn{2}{|c|}{ Anaemic $(n 34,16.7 \%)$} & \multicolumn{2}{|c|}{ Normal ( $n 169,83.3 \%)$} & \multicolumn{2}{|c|}{ Unadjusted } & \multicolumn{2}{|c|}{ Anaemic $(n 100,34 \cdot 2 \%)$} & \multicolumn{2}{|c|}{ Normal (n 192, 65.8\%) } & \multicolumn{2}{|c|}{ Unadjusted } \\
\hline & $n$ & $\%$ & $n$ & $\%$ & OR & $95 \% \mathrm{Cl}$ & $n$ & $\%$ & $n$ & $\%$ & OR & $95 \% \mathrm{Cl}$ \\
\hline \multicolumn{13}{|c|}{ Intake of red meat } \\
\hline Frequently & 18 & 52.9 & 85 & $50 \cdot 3$ & 1.0 & Ref. & 52 & $52 \cdot 0$ & 110 & $57 \cdot 3$ & 1.0 & Ref. \\
\hline Infrequently & 14 & $41 \cdot 2$ & 72 & $42 \cdot 6$ & 0.9 & $0.4,1.9$ & 42 & $42 \cdot 0$ & 67 & 34.9 & 1.3 & $0.8,2 \cdot 2$ \\
\hline Never & 2 & 5.9 & 12 & $7 \cdot 1$ & 0.8 & $0.5,3.8$ & 6 & 6.0 & 15 & $7 \cdot 8$ & 0.8 & $0 \cdot 3,2 \cdot 3$ \\
\hline \multicolumn{13}{|c|}{ Intake of green vegetables } \\
\hline Frequently & 22 & 64.7 & 99 & $58 \cdot 6$ & 1.0 & Ref. & 68 & 68.0 & 126 & 65.6 & 1.0 & Ref. \\
\hline Infrequently & 9 & 26.5 & 57 & 33.7 & 0.7 & $0.3,1.6$ & 26 & $26 \cdot 0$ & 57 & 29.7 & 0.8 & $0.5,1.5$ \\
\hline Never & 3 & 8.8 & 13 & 7.7 & $1 \cdot 1$ & $0.2,3.9$ & 6 & 6.0 & 9 & 4.7 & 1.2 & $0.4,3.6$ \\
\hline \multicolumn{13}{|c|}{ Intake of tea/coffee } \\
\hline Frequently & 12 & $35 \cdot 3$ & 38 & 22.5 & 1.0 & Ref. & 26 & $26 \cdot 0$ & 53 & $27 \cdot 6$ & 1.0 & Ref. \\
\hline Infrequently & 5 & 14.7 & 24 & $14 \cdot 2$ & 0.6 & $0.2,2 \cdot 1$ & 20 & $20 \cdot 0$ & 23 & 12.0 & 1.7 & $0 \cdot 7,3 \cdot 7$ \\
\hline Never & 17 & $50 \cdot 0$ & 107 & 63.3 & 0.5 & $0.2,1 \cdot 1$ & 54 & 54.0 & 116 & 60.4 & 0.9 & $0.5,1.7$ \\
\hline \multicolumn{13}{|l|}{ Intake of laban } \\
\hline Frequently & 17 & $50 \cdot 0$ & 87 & 51.5 & $1 \cdot 0$ & Ref. & 54 & $54 \cdot 0$ & 109 & $56 \cdot 8$ & 1.0 & Ref. \\
\hline Infrequently & 14 & $41 \cdot 2$ & 55 & 32.5 & 1.3 & $0.6,2 \cdot 8$ & 29 & 29.0 & 58 & $30 \cdot 2$ & 1.0 & $0.6,1.7$ \\
\hline Never & 3 & 8.8 & 27 & $16 \cdot 0$ & 0.6 & $0 \cdot 1,2 \cdot 1$ & 17 & 17.0 & 25 & 13.0 & 1.4 & $0 \cdot 7,2 \cdot 7$ \\
\hline \multicolumn{13}{|c|}{ Intake of soft drinks } \\
\hline Frequently & 15 & $44 \cdot 1$ & 75 & $44 \cdot 4$ & $1 \cdot 0$ & Ref. & 47 & 47.0 & 87 & $45 \cdot 3$ & 1.0 & Ref. \\
\hline Infrequently & 10 & 29.4 & 43 & $25 \cdot 4$ & $1 \cdot 2$ & $0.5,2.8$ & 28 & 28.0 & 50 & 26 & 1.0 & $0.5,1.8$ \\
\hline Never & 9 & 26.5 & 51 & $30 \cdot 2$ & 0.8 & $0.4,2.2$ & 25 & $25 \cdot 0$ & 55 & 28.6 & 0.8 & $0.5,1.5$ \\
\hline \multicolumn{13}{|c|}{ Intake of fruit juices } \\
\hline Frequently & 7 & $20 \cdot 6$ & 87 & 51.5 & 1.0 & Ref. & 20 & $20 \cdot 0$ & 80 & 41.7 & 1.0 & Ref. \\
\hline Infrequently & 10 & 29.4 & 33 & 19.5 & 3.7 & $1 \cdot 3,10 \cdot 1$ & 29 & 29.0 & 49 & $25 \cdot 5$ & $2 \cdot 3$ & $1 \cdot 2,4 \cdot 6$ \\
\hline Never & 17 & $50 \cdot 0$ & 49 & 29.0 & $4 \cdot 3$ & $1 \cdot 6,11 \cdot 2$ & 51 & 51.0 & 63 & $32 \cdot 8$ & 3.2 & $1 \cdot 7,5 \cdot 9$ \\
\hline \multicolumn{13}{|l|}{ Intake of eggs } \\
\hline Frequently & 17 & $50 \cdot 0$ & 66 & 39.1 & 1.0 & Ref. & 44 & $44 \cdot 0$ & 90 & $46 \cdot 9$ & 1.0 & Ref. \\
\hline Infrequently & 15 & 44.1 & 76 & $45 \cdot 0$ & 0.7 & $0.3,1.6$ & 44 & 44.0 & 82 & $42 \cdot 7$ & 1.0 & $0.6,1.8$ \\
\hline Never & 2 & 5.9 & 27 & $16 \cdot 0$ & 0.3 & $0.1,1.3$ & 12 & 12.0 & 20 & $10 \cdot 4$ & 1.2 & $0.5,2.7$ \\
\hline
\end{tabular}

Ref., reference category.

Frequently $=5-7 \mathrm{~d} /$ week; infrequently $=2-4 \mathrm{~d} /$ week; never $=0-1 \mathrm{~d} /$ week 
Table 4 Multivariate linear regression model results for associations between sociodemographic, family history and diet and anaemia in male adolescents (aged 13-18 years) in Riyadh, Kingdom of Saudi Arabia ( $n$ 203)

\begin{tabular}{lccc}
\hline Variable & Adjusted OR & $95 \% \mathrm{Cl}$ & $P$ value \\
\hline $\begin{array}{l}\text { Family history of Fe-deficiency anaemia } \\
\text { No }\end{array}$ & 1.0 & & \\
Yes & 4.7 & $1.7,12.2$ & 0.04 \\
Intake of fruit juices & & & \\
$\quad$ Frequently & 1.0 & Ref. & - \\
Infrequently & 3.7 & $1.3,10.0$ & 0.02 \\
$\quad$ Never & 3.5 & $1.4,9.5$ & 0.03 \\
Age group & & & \\
17-18 years & 1.0 & Ref. & - \\
15-16 years & 0.8 & $0.2,3.2$ & 0.86 \\
$13-14$ years & 3.1 & $1.2,9.3$ & 0.04 \\
\hline
\end{tabular}

Ref., reference category.

Frequently $=5-7 \mathrm{~d} /$ week; infrequently $=2-4 \mathrm{~d} /$ week; never $=0-1 \mathrm{~d} /$ week.

Table 5 Multivariate linear regression model results for associations between sociodemographic, family history, diet intake and anaemia in female adolescents (aged 13-18 years) in Riyadh, Kingdom of Saudi Arabia ( $n$ 292)

\begin{tabular}{lccc}
\hline Variable & Adjusted OR & $95 \% \mathrm{Cl}$ & $P$ value \\
\hline $\begin{array}{l}\text { Family history of Fe-deficiency anaemia } \\
\text { No }\end{array}$ & 1.0 & & \\
$\quad$ Yes & 3.4 & $1.5,7.6$ & 0.004 \\
BMl category & & & \\
$\quad$ Normal & 1.0 & Ref. & - \\
$\quad$ Underweight & 1.4 & $0.7,2.7$ & 0.38 \\
Overweight & 3.0 & $1.4,6 \cdot 1$ & 0.002 \\
$\quad$ Obese & 1.2 & $0.4,3.4$ & 0.61 \\
Intake of fruit juices & & & \\
Frequently & 1.0 & Ref. & - \\
Infrequently & 1.8 & $0.8,3 \cdot 6$ & 0.14 \\
$\quad$ Never & 2.6 & $1.4,5 \cdot 1$ & 0.004 \\
Type of housing & & & \\
$\quad$ Villa (modern house) & 1.0 & Ref. & - \\
Apartment & 2.0 & $1.1,3.8$ & 0.01 \\
Small house & 2.5 & $1.2,5.3$ & 0.01 \\
\hline
\end{tabular}

Ref., reference category.

Frequently $=5-7 \mathrm{~d} /$ week; infrequently $=2-4 \mathrm{~d} /$ week; never $=0-1 \mathrm{~d} /$ week.

for socio-economic conditions; Table 4). In females, a family history of IDA (OR $=3 \cdot 4 ; 95 \%$ CI $1 \cdot 5,7 \cdot 6)$, being overweight $(\mathrm{OR}=3.0 ; 95 \%$ CI 1.4, 6.1), no intake of fresh juices $(\mathrm{OR}=2 \cdot 6 ; 95 \%$ CI $1 \cdot 4,5 \cdot 1)$ and living in an apartment or a small house $(\mathrm{OR}=2 \cdot 1 ; 95 \% \mathrm{CI} 1 \cdot 1,3.8$ and $\mathrm{OR}=2 \cdot 6 ; 95 \% \mathrm{CI}$ $1 \cdot 2,5 \cdot 3$, respectively) were significantly associated with anaemia (after adjusting for age; Table 5).

\section{Discussion}

Our community-based research was conceived and designed with the objective to determine the prevalence and correlates of anaemia in male and female adolescents. Our findings revealed that young age, being overweight, having a positive family history of IDA, low socio-economic status and lack of intake of fruit juices were significantly associated with anaemia in adolescents in KSA. The prevalence rates of
$16.7 \%$ and $34.0 \%$ in male and female adolescents, respectively, are in line with previous studies conducted in the Kingdom and other Gulf countries, which reported anaemia to be higher among female adolescents (22.4-40.0\%) than in males $(12 \cdot 8-18 \cdot 4 \%)^{(2,5-7,9)}$. It is suggested that in males the achievement of adult testosterone concentration is associated with an increase in erythropoiesis ${ }^{(21)}$; therefore, the lower prevalence of anaemia among pubertal/ adolescent boys can be explained by the physiological rise in $\mathrm{Hb}$ concentration caused by sexual maturation as well as by decreasing requirements after completion of the growth spurt $^{(22,23)}$. This fact is supported in our study by showing the 13-14 year age category being at risk of developing anaemia as compared with older age groups (15 and above years). The mean $\mathrm{Hb}$ among 13-14-year-old boys was $12 \cdot 4 \mathrm{mg} / \mathrm{dl}$, whereas among boys aged 15-16 years and $17-18$ years it was $13.64 \mathrm{mg} / \mathrm{dl}$ and $14.24 \mathrm{mg} / \mathrm{dl}$, respectively. In contrast, among girls, occurrence of menarche and menstrual irregularities may halt an expected age-related increase in $\mathrm{Hb}$ concentration ${ }^{(24)}$. In our study, about $80 \%$ of females stated having menarche at 12-13 years; hence the higher (34.2\%) prevalence of anaemia among females could be due to earlier pubertal onset or menarche.

Several studies have established overweight and malnourishment as important factors causing anaemia ${ }^{(25,26)}$. Our results are in accordance with previous studies; however, this association was significant among females only. Recently, the role of inflammatory processes in causing anaemia among overweight/obese people has been highlighted $^{(26)}$. Overweight/obesity is considered a chronic inflammatory process because macrophages in adipose tissues synthesize pro-inflammatory cytokines including IL-6 and TNF- $\alpha$. These cytokines lead to increased hepcidin release/synthesis from the liver and adipocytes. Hepcidin causes degradation of ferroportin, which is a cellular Fe exporter at the baso-lateral membrane of duodenal enterocytes and macrophages, resulting in inhibition of Fe flow into the plasma from dietary Fe-absorbing enterocytes and Fe-recycling macrophages $^{(27,28)}$. This leads to impaired dietary Fe repletion and reduced $\mathrm{Fe}$ stores causing anaemia of chronic inflammation condition. Obesity is highly prevalent among Saudi children and adolescents; and with the help of above explanation, we can easily anticipate the burden of anaemia among adolescents. In addition, sedentary lifestyle, consumption of highly caloric foods deficient in Fe/essential nutrients and the greater need for Fe intake associated with body weight further increase the risk of anaemia among those who are obese and overweight ${ }^{(29)}$. The reason for not finding a similar association among males could be the small number $(n 7)$ of male participants in this specific category. In addition, the food habits of the Saudi population have changed drastically from the traditional diet, which consisted of dates, milk, rice, fresh vegetables and fish, being replaced with junk foods and less green vegetables and fruits ${ }^{(30)}$. 
Intakes of fruits and vegetables make an essential part of a healthy diet because they protect from and prevent several chronic diseases ${ }^{(31)}$. One important component of fruit juices is ascorbic acid (vitamin C). Ascorbic acid, which is an antioxidant, enhances the absorption of nonhaem $\mathrm{Fe}$ in the body ${ }^{(32)}$. An experimental study conducted by Kim et al. found ascorbic acid could counteract the inhibitory effect of polyphenolic compounds and hence enhance the transepithelial $\mathrm{Fe}$ transportation ${ }^{(33)}$. The average daily requirement for ascorbic acid for males and females ranges from 65 to $75 \mathrm{mg}^{(34)}$. According to one Spanish study, various fruit juices contain high quantities of ascorbic acid (352-840 mg/litre) which is several times more than the average daily requirement $(65-75 \mathrm{mg})^{(35)}$. We are limited in commenting on the quantity of vitamin $\mathrm{C}$ consumed, as we did not collect the information on quantity of fruit juice consumed by the adolescents. In addition to the above, there may be different reasons explaining the association between fruit juice and anaemia; these individuals may be generally following a healthy lifestyle, or may be more aware about quality diet perhaps because of higher socio-economic class (although in KSA a one-quarter size fruit juice pack is 1.50 SR only). The last reason is supported by our results which found that adolescents living in villas consumed fruit juice more frequently than those living in apartments; however, these findings were statistically insignificant in males $(P=0 \cdot 08)$ and significant in female adolescents only $(P<0.05)$.

We found a strong association between a positive family history of IDA and anaemia in adolescents $(\mathrm{OR}=2 \cdot 64$; $95 \%$ CI $1.27,5.09)$. A family history of IDA among mothers, siblings, spouses or fathers increased the risk by three- to sixfold ${ }^{(36)}$. Hence, if anaemia is diagnosed even in a single member of the family, all other members should undergo screening to identify the cause and prevent future grave consequences. Various studies are available suggesting that poor household environment, high crowding index, poor hygiene and low educational background all contribute towards anaemia ${ }^{(19)}$. Our participants were adolescents and therefore their age corresponded to their educational level, so using any one variable in the model was logical. Bilharziasis, a disease once prevalent in KSA, mostly in the south region ${ }^{(37)}$, was significant in univariate analysis only. Bilharziasis mostly occurs due to unawareness and unhygienic conditions ${ }^{(37)}$; however, because of small numbers we were not able to find it significant in the multivariate models.

The selection of participants from different types of randomly selected households makes our results generalizable to urban areas of KSA. Galobardes et al. have published a comprehensive glossary of indicators of socioeconomic position, which include ownership of one's house and household conditions ${ }^{(38)}$. Riyadh, being the capital of KSA, has all types of social classes residing in it. The housing is basically divided into four types: (i) the palaces owned only by the members of the royal family and the rich sheikhs; (ii) the villas owned by the higher and the upper middle class; (iii) the old traditional houses (small houses) mostly owned by middle-class old Saudi families; and (iv) apartments owned mostly by the working class. The participants in our study were adolescents (school-going and unemployed); therefore, 'type of house' as indicating socio-economic status was the best choice. Moreover, we found that socio-economic status did not affect the number of adolescents participating in the study $(P=0.94)$. We encountered some refusals; however, it did not affect our sample size. The refusal was because of various reasons: the mobility of females is restricted in KSA and they cannot come alone to the health centre; adolescents' school timings clashing with the health centre timings; and some refused because of extreme hot weather.

This was a community-based study and included adolescents belonging to all three age groups (13-14 years, 15-16 years and 17-18 years). There are some limitations with the study. First, our diagnosis for anaemia was based on single measure of blood $\mathrm{Hb}$ level and other blood indices (Table 1) and we were limited in measuring serum ferritin, which is essential for labelling anaemia as IDA; hence there may be some misclassification bias. Second, the study was conducted in Riyadh alone, which is an urban city; therefore the results are representing only the urban settlements of KSA. Third, food items like meat, eggs and vegetables are considered good sources of Fe; however, we did not collect detailed history about the quantity or portion size of different dietary items, which may be the reason for not finding an association. In addition, we did not take information on Fe supplements or multivitamins, which may have affected the blood $\mathrm{Hb}$ level. Fourth, information related to age, diet and medical history was based on verbal responses; hence information bias related to exposures cannot be ruled out.

\section{Conclusion}

Our results conclude that the prevalence of anaemia in KSA is as high as before. According to the WHO, a $16-34 \%$ prevalence of anaemia among adolescents places KSA among countries with anaemia as a moderate category public health problem. This problem is more prevalent among female adolescents. The significantly associated factors like young age, positive family history of IDA, overweight, lack of fresh juice intake and low socioeconomic status are apparently diverse and it may be a challenge to deal with all them together. Therefore, if the focus is on key factors like positive family history of IDA and low socio-economic status, we may be able to reduce the prevalence of anaemia. Families with poor socioeconomic status are usually less aware and unconcerned about the quality of their diet (lack of fruits and vegetables in the diet), which may lead to problems like overweight and obesity, further aggravating the problem. Hence, early 
identification and family screening is important. We can utilize various methods of screening; one may be the mass screening programmes conducted on an annual basis. Another method can be screening using the school health programmes and then reaching out to other family members. A recent review on the health system in $\mathrm{KSA}^{(39)}$ documented the existence of school health units; however, we are not sure about their functioning.

Simple interventions, like health education, can create awareness and help in adopting healthy quality diet to prevent anaemia. The WHO has adopted various strategies that range from single interventions like 'weekly iron and folic acid supplementation' to a multi-sectoral approach $^{(40)}$. One good example of the multi-sectoral approach is the control of anaemia in Nicaragua ${ }^{(41)}$. This programme was successful as institutes both at central level and local level along with communities participated. There was coordination between different sectors like health, education, food, social services, information and technology, and even international agencies. KSA should also adopt a multi-sector community-based approach for the prevention and control of anaemia. It should formulate a policy for the fortification of food items along with a health education component implemented at school level (both religious and ordinary). Participation of social agencies, religious scholars and parents can make such initiatives successful.

\section{Acknowledgements}

Acknowledgements: The authors thank the Research Chairs Program - King Saud University and the Ministry of Health for their unlimited support. They also would like to extend thanks to the PHCC staff for their efforts and cooperation in conducting this study. Financial support: This work was funded by Ministry of Health, KSA. Conflict of interest: None. Authorship: A.-J.M.A. and T.A.M.K. formulated the research question and the methodolgy of the study. A. Alsharif, H.A.M. and A. Aldiris helped in coordinating with the Ministry of Health and conduction of the fieldwork at different PHCC. A.G.M. helped in questionnaire formulation and data collection. A.K. and S.A.S. worked on the analysis and the write-up of the study. All authors reviewed the manuscript and assisted in its completion. Ethical disclosure: This study was conducted according to the guidelines laid down in the Declaration of Helsinki and all procedures involving human subjects were approved by the Institutional Review Board, King Saud University. Written informed consent was obtained from all subjects.

\section{References}

1. World Health Organization (2008) Worldwide Prevalence of Anaemia 1993-2005. WHO Global Database on Anaemia. Geneva: WHO; available at http://whqlibdoc.who.int/ publications/2008/9789241596657_eng.pdf.
2. Jackson TR \& Al-Mousa Z (2000) Iron deficiency is a more important cause of anemia than hemoglobinopathies in Kuwaiti adolescent girls. J Nutr 130, 1212-1216.

3. Al-Sharbatti SS, Al-Ward JN \& Al-Timimi JD (2003) Anemia among adolescents. Saudi Med J 24, 189-194.

4. Barakat-Haddad C (2013) Prevalence of high blood pressure, heart disease, thalassemia, sickle-cell anemia, and irondeficiency anemia among the UAE adolescent population. J Environ Public Health 2013, 680631.

5. Abou-Zeid AH, Abdel-Fattah MM, Al-Shehri AS et al. (2006) Anemia and nutritional status of schoolchildren living at Saudi high altitude area. Saudi Med J 27, 862-869.

6. Madani AK \& Kumosani AT (2001) Micronutrient status in Saudi Arabia. Babrain Med Bull 23, 1-11.

7. Al-Shehri S (1996) Health Profile of Saudi Adolescent Schoolgirls. Riyadh: Presidency of Girls Education, Health Affairs Directorate.

8. El-Hazmi MA \& Warsy AS (2001) Normal reference values for hematological parameters, red cell indices, $\mathrm{Hb}$ A2 and $\mathrm{Hb} \mathrm{F}$ from early childhood through adolescence in Saudis. Ann Saudi Med 21, 165-169.

9. Abalkhail B \& Shawky S (2002) Prevalence of daily breakfast intake, iron deficiency anemia and awareness of being anemic among Saudi school students. Int J Food Sci Nutr 53, 519-528.

10. Mesías M, Seiquer I \& Navarro MP (2013) Iron nutrition in adolescence. Crit Rev Food Sci Nutr 53, 1226-1237.

11. Chan LN \& Mike LA (2014) The science and practice of micronutrient supplementations in nutritional anemia: an evidence-based review. JPEN J Parenter Enteral Nutr 38, 656-672.

12. Rahman A \& Chowdhury S (2007) Determinants of chronic malnutrition among preschool children in Bangladesh. J Biosoc Sci 39, 161-173.

13. Aderibigbe OR, Pisa PT, Vorster HH et al. (2014) The relationship between iron status and adiposity in women from developing countries: a review. Crit Rev Food Sci Nutr $\mathbf{5 4}$ 553-560.

14. Cao C \& O'Brien KO (2013) Pregnancy and iron homeostasis: an update. Nutr Rev 71, 35-51.

15. Chen MH, Su TP, Chen YS et al. (2013) Association between psychiatric disorders and iron deficiency anemia among children and adolescents: a nationwide populationbased study. BMC Psychiatry 13, 161.

16. Zofková I, Nemcikova P \& Matucha P (2013) Trace elements and bone health. Clin Chem Lab Med 51, 1555-1561.

17. Falkingham M, Abdelhamid A, Curtis P et al. (2010) The effects of oral iron supplementation on cognition in older children and adults: a systematic review and meta-analysis. Nutr J 9, 4.

18. Al-Hazzaa HM, Abahussain NA, Al-Sobayel HI et al. (2011) Physical activity, sedentary behaviors and dietary habits among Saudi adolescents relative to age, gender and region. Int J Behav Nutr Phys Act 8, 140.

19. Kingdom of Saudi Arabia, Ministry of Economy \& Planning, Central Department of Statistics \& Information, Department of Analysis \& Reports (2010) Statistical Year Book 46 (14311432). http://www.cdsi.gov.sa/yb46/ (accessed June 2014).

20. UNICEF, United Nations University \& World Health Organization (2012) Iron Deficiency Anaemia. Assessment, Prevention and Control. A Guide for Programme Managers. WHO/NHD/01.3. Geneva: WHO; available at http://www. who.int/nutrition/publications/en/ida_assessment_prevention_ control.pdf.

21. Thomsen K, Riis B, Krabbe S et al. (1986) Testosterone regulates the haemoglobin concentration in male puberty. Acta Paediatr Scand 75, 793-796.

22. De Andrade Cairo RC, Rodriques Silva L, Carneiro Bustani N et al. (2014) Iron deficiency anemia in adolescents; a literature review. Nutr Hosp 29, 1240-1249. 
23. Milman N (2011) Anemia - still a major health problem in many parts of the world!. Ann Hematol 90, 369-377.

24. Rigon F, De Sanctis V, Bernasconi S et al. (2012) Menstrual pattern and menstrual disorders among adolescents: an update of the Italian data. Ital J Pediatr 38, 38.

25. Nead KG, Halterman JS, Kaczorowski JM et al. (2004) Overweight children and adolescents: a risk group for iron deficiency. Pediatrics 114, 104-108.

26. McClung JP \& Karl JP (2009) Iron deficiency and obesity: the contribution of inflammation and diminished iron absorption. Nutr Rev 67, 100-104.

27. Collins JF, Wessling-Resnick M \& Knutson MD (2008) Hepcidin regulation of iron transport. J Nutr 138, 2284-2288.

28. Sanad M, Osman M \& Gharib A (2011) Obesity modulate serum hepcidin and treatment outcome of iron deficiency anemia in children: a case control study. Ital J Pediatr 37, 34 .

29. Manios Y, Moschonis G, Chrousos GP et al. (2013) The double burden of obesity and iron deficiency on children and adolescents in Greece: the Healthy Growth Study. J Hum Nutr Diet 26, 470-478.

30. Al-Othaimeen AL, Al-Nazha M \& Osman AK (2007) Obesity: an emerging problem in Saudi Arabia. Analysis of data from the National Nutrition Survey. East Mediterr Health J 13, 441-448.

31. World Health Organization (2015) Healthy diet. Fact sheet no. 394. http://www.who.int/mediacentre/factsheets/fs394/ en/ (accessed February 2015)

32. Ozgur MU \& Sungur S (1995) Third order derivative spectrophotometric determination of ascorbic acid in fruits and vegetables. Talanta 42, 1631-1640.
33. Kim EY, Ham SK, Bradke D et al. (2011) Ascorbic acid offsets the inhibitory effect of bioactive dietary polyphenolic compounds on transepithelial iron transport in Caco-2 intestinal cells. $J$ Nutr 141, 828-834.

34. National Institutes of Health, Office of Dietary Supplements (2011) Vitamin C fact sheet for consumers. http://ods.od.nih. gov/factsheets/VitaminC-QuickFacts/ (accessed July 2014).

35. Rodríguez-Bernaldo de Quirós A, Fernández-Arias M \& López-Hernández J (2009) A screening method for the determination of ascorbic acid in fruit juices and soft drinks. Food Chem 116, 509-512.

36. Al-Quaiz MJ (2001) Iron deficiency anemia. Saudi Med J 22, 490-496.

37. Lotfy WM \& Alsaqabi SM (2010) Human schistosomiasis in the Kingdom of Saudi Arabia: a review. J Med Res Inst 31, 1-6.

38. Galobardes B, Shaw M, Lawlor DA et al. (2006) Indicators of socioeconomic position (part 1). J Epidemiol Community Health 60, 7-12.

39. Almalki M, Fitzgerald G \& Clark M (2011) Health care system in Saudi Arabia: an overview. East Mediterr Health J 17, 784-793.

40. World Health Organization (2011) Prevention of Iron Deficiency Anemia in Adolescents. Role of Weekly Iron and Folic Acid Supplementation. New Delhi: WHO Regional Office for South-East Asia; available at http://apps.searo. who.int/PDS_DOCS/B4770.pdf?ua=1.

41. Mora AJ (2007) Integrated anemia control strategy has significantly reduced anemia in women and children in Nicaragua. http://www.micronutrient.org/CMFiles/MI\%20Around \%20the\%20World/Americas/Nicaragua_Anemiacontrolprog finalrpt.pdf (accessed April 2015). 